\title{
Albert Einstein and Relativity
}

\author{
Kamal B Khatri \\ Department of Physics,PN Campus,Pokhara,Email:kmlphy_123@yahoo.com
}

Albert Einstein was born in Germany in 1879.In his life, Einstein spent his most time in Germany, Italy, Switzerland and USA.He is also a Nobel laureate and worked mostly in theoretical physics. Einstein is best known for his theories of special and general relativity. I will not be wrong if I say Einstein a deep thinker, a philosopher and a real physicist. The philosophies of Henri Poincare, Ernst Mach and David Hume influenced Einstein's scientific and philosophical outlook.

Einstein at the age of 4 , his father showed him a pocket compass, and Einstein realized that there must be something causing the needle to move, despite the apparent 'empty space'. This shows Einstein's curiosity to the space from his childhood. The space of our intuitive understanding is the 3-dimensional Euclidean space. By the age of 12, he had learned Euclidean geometry (it is based on the axiom that between any two points a straight line may be produced). But the path followed by a particle in space may not be always straight line; which is nonEuclidean space. German mathematician Wilhelm Karl Joseph Killing contributed to develop nonEuclidean geometry.

One of the philosophers who influenced Einstein is Austrian philosopher- Ernst Mach. Lets see how Einstein got influenced by Mach! According to Mach's principle, the measure of inertia of a body depends on the existence of the background in such a way that in the absence of any background the measure vanishes. To see it, let us consider a body in an otherwise empty universe where the force on the body, $\mathrm{F}=0=\mathrm{m} . \mathrm{a} \Rightarrow \mathrm{m}=\mathrm{o}$, because there is no background to measure the acceleration i.e. ' $a$ ' is indeterminate! This means in the absence of the background the measure of inertia vanishes. This implies that inertia is not the absolute property, it is also relative. This fact shocked Einstein so that he changed his outlook to space. Later, Einstein became the follower of Mach and his nascent concept helped him to enter the world of relativity.

In 1905, Einstein propounded the "Theory of Special Relativity". This theory shows the observed independence of the speed of light on the observer's state of motion. Einstein deduced from his concept of special relativity the twentieth century's best known equation, $\mathrm{E}=\mathrm{m} \mathrm{c} 2$. This equation suggests that tiny amounts of mass can be converted into huge amounts of energy which in deed, became the boon for the development of nuclear power.

Einstein realized that the principle of special relativity could be extended to gravitational fields. Since Einstein believed that the laws of physics were local, described by local fields, he concluded from this that space time could be locally curved. This led him to study Riemannian geometry; a kind of geometry where the curvature changes from point to point. For this, Einstein visited Zurich mathematician Marcel Grossmann, Italian mathematician Tullio Levi-civet, and studied the usefulness of tensor. By late 1915, he had published "General theory of relativity" in the form in which it is used today. This theory is based on equivalence principle and non-Euclidean geometry; and it has two fundamental laws - the Einstein equations which describe how space curves and the geodesic equation which describes how particles move. Moreover, this theory explains gravitation as distortion of the structure of space-time by matter, affecting the inertial motion of other matter .In other words, this theory says that gravity is really a curvature of space.But,it appeared at first through involved only in very abstruse examples such as the behavior of light around the sun and in eclipse. Later, British astrophysicist Eddington measured the bending of light and it was a typical evidence of the theory .This spectacular proof brought Einstein into public eye. Many scientists praised the theory of general relativity as the 'greatest feat of human 
thinking about nature'; and as 'probably the greatest scientific discovery ever made'. Later, Einstein applied this theory to model the structure of the universe as a whole.

Following his research on general relativity, Einstein entered into a series of attempts to generalize his theory of gravitation. In 1950, he described his 'unified field theory' in an article entitled "On the generalized theory of gravitation". Although he continued to be lauded for his work, Einstein became increasingly isolated in his research, and his efforts were ultimately unsuccessful. Because of his fragile health, Einstein had to stop his ceaseless effort. In his pursuit of a unification of the fundamental forces, Einstein ignored some mainstream development in physics, most notably the strong and weak nuclear forces, which were not well understood until many years after his death. Mainstream physics in turn, largely ignored Einstein's approaches to unification .Einstein's dream of unifying other laws of physics with gravity motivates modern quests for a 'theory of everything' and in particular 'String theory'. "IMAGINATION IS MORE IMPORTANT THAN KNOWLEDGE", SAID EINSTEIN. Really, Einstein has shown the path to imagine more and more for new generation!!!

\section{References:}

Principles \& Applications of general theory of relativity by Steven Weinberg

The Early Universe by Kolb Turner (Year ?)

Einstein encyclopedia, publisher and year 